\title{
On the Regional Enlarged Observability for Linear Parabolic Systems
}

\author{
Hayat Zouiten, Ali Boutoulout and Fatima Zahrae El Alaoui \\ TSI Team, MACS Laboratory, Faculty of Sciences, Moulay Ismail University, Meknes, Morocco
}

Received: November 10, 2016 / Accepted: December 08, 2016 / Published: March 25, 2017.

\begin{abstract}
In this paper, we study the notion of the enlarged observability for distributed parabolic systems, where the aim is to reconstruct the initial state between two prescribed profiles $P_{1}$ and $P_{2}$ in an internal subregion $\omega$ of the evolution domain $\Omega$. We give some definitions and properties of this concept, and then we solve the problem of the reconstruction of initial state using the Hilbert Uniqueness Method (HUM). This leads to several interesting results which are performed through numerical example and simulations.
\end{abstract}

Key words: Distributed systems, Parabolic systems, Enlarged observability, HUM Approach.

\section{Introduction}

Distributed parameter systems can be used to describe many real phenomena. They consist of a set of notions as controllability, observability, stability, etc, which lead to various methods of analysis and applications. The observability is one of the most fundamental issues associated with the analysis and control of systems. It concerns the real identification of the trajectory, or in an equivalent way, the reconstruction of the initial state or gradient from the measurements taken of the system by the means of tools called sensors. This concept has been the subject of a good deal of research (see [2], [3], [5]). Afterward, the notion of regional observability was introduced and interesting results have been obtained (see [1], [4]), in particular, the possibility to reach a state only in a subregion $\omega$ of the system evolution domain $\Omega$. This concept was extended to the case where $\omega$ is a part of the boundary $\partial \Omega$ of $\Omega$ (see [10]).

In this work, we'll develop the concept of enlarged observability also called constrained observability for

Corresponding author: Ali Boutoulout, TSI Team, MACS Laboratory, Faculty of Sciences, Moulay Ismail University, Meknes, Morocco. linear parabolic systems, where the goal is to reconstruct the initial state between two prescribed functions only in a critical internal subregion $\omega$ of $\Omega$. The principal reason behind introducing this notion is that it provides a means to deal with some physical problems, where the reconstructed state is required only to be between two profiles. This is the case, for example of a biological reactor (Figure. 1) in which the concentration regulation of a substrate at the bottom of the reactor is expected to be between two levels (see [9]).

The paper is organized as follows. Section 2, we introduce the notion of the enlarged internal observability for distributed linear parabolic systems. Section 3, concerns a reconstruction approach using an extension of the Hilbert Uniqueness Method (HUM) developed by Lions (see [7], [8]). In the last section, we present the obtained algorithm with numerical example and simulations.

\section{Problem Statement}

Let $\Omega$ be an open bounded domain in $\mathbb{R}^{n}$ $(n=1,2,3)$, with a boundary $\partial \Omega$ which is regular enough. For $T \succ 0$, let $Q=\Omega \times] 0, T[$ and 


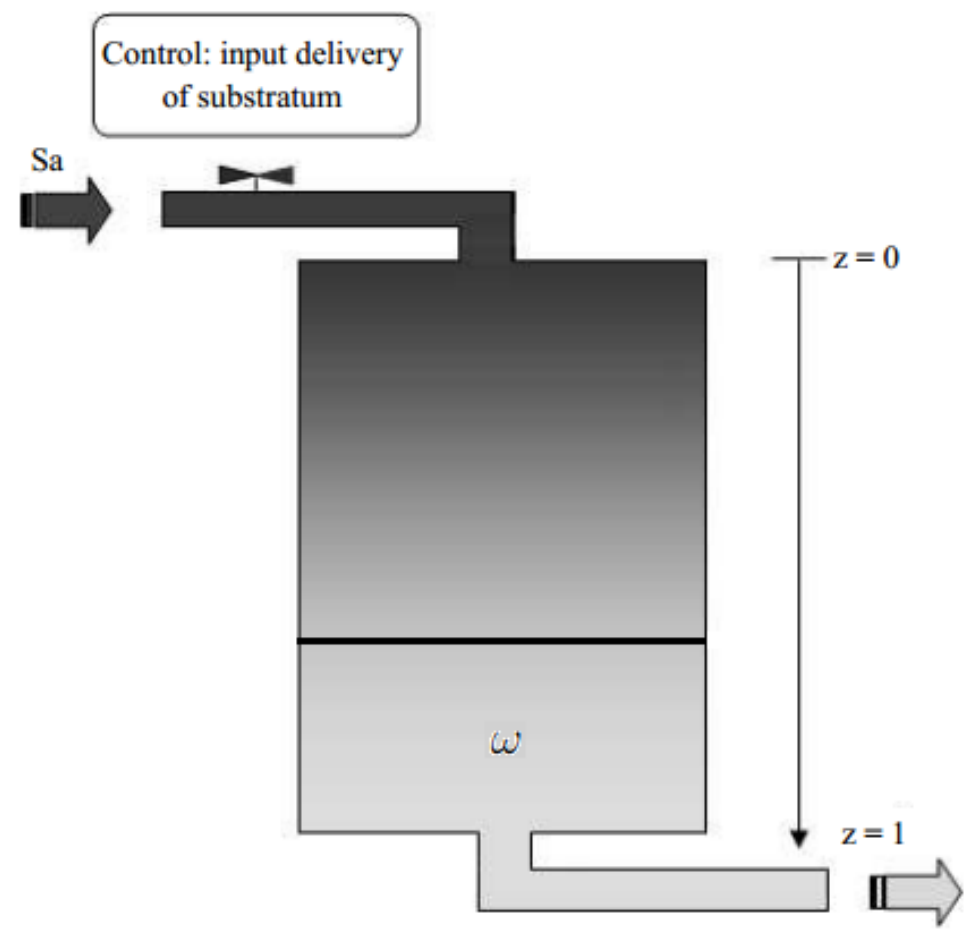

Fig. 1 Regulation of the concentration flux of the substratum at a bottom of the reactor.

$\Sigma=\partial \Omega \times] 0, T[$. Then we consider the following parabolic equation

$$
\left\{\begin{array}{lll}
\frac{\partial y(x, t)}{\partial t}=A y(x, t) & \text { in } & Q \\
y(x, 0)=y_{0}(x) & \text { in } & \Omega \\
y(\xi, t)=0 & \text { on } & \Sigma,
\end{array}\right.
$$

where $\mathrm{A}$ is a second-order linear differential operator with compact resolvent which generates a strongly continuous semigroup $(S(t))_{t \geq 0}$ on the state space $L^{2}(\Omega)$. Without loss of generality we denote $y(x, t):=y(t)$. We suppose that $y_{0} \in L^{2}(\Omega)$ then the system (1) admits a unique solution $y($.$) in$ $L^{2}(\Omega \times] 0, T[) \quad$ (see [6]). The measurements are obtained by the output function given by

$$
z(t)=C y(., t), \quad t \in[0, T],
$$

where $C \in \mathcal{L}\left(\mathrm{L}^{2}(\Omega), \mathbb{R}^{q}\right)$ is called the observation operator, linear depending on the structure and the number $\mathrm{q}$ of the considered sensors. The observation space is $O=\mathrm{L}^{2}\left(0, T ; \mathbb{R}^{q}\right)$.

Since the system (1) is autonomous, the output function can be expressed by

$$
z(t)=C S(t) y_{0}, \quad t \in[0, \mathrm{~T}] .
$$

We define the operator

$$
\begin{aligned}
K: \mathrm{L}^{2}(\Omega) & \rightarrow O \\
z & \mapsto C S(.) z,
\end{aligned}
$$

which is linear bounded with the adjoint $K^{*}$ given by

$$
\begin{aligned}
K^{*}: O & \rightarrow \mathrm{L}^{2}(\Omega) \\
z & \mapsto \int_{0}^{T} S^{*}(t) C^{*} z(t) d t .
\end{aligned}
$$

Let $\omega$ be a subregion of $\Omega$ with a positive Lebesgue measure. Let $\chi_{\omega}$ be the restriction function defined by

$$
\begin{aligned}
\lambda_{\omega}: \mathrm{L}^{2}(\Omega) & \rightarrow \mathrm{L}^{2}(\omega) \\
y & \mapsto \lambda_{\omega} y=y_{\mid \omega},
\end{aligned}
$$


with the adjoint $\chi_{\omega}^{*}$ given by

$$
\chi_{\omega}^{*} y= \begin{cases}y & \text { in } \omega \\ 0 & \text { in } \Omega \backslash \omega .\end{cases}
$$

We recall that a sensor is conventionally defined by a couple $(D, f)$, where $D$ is its spatial support represented by a nonempty part of $\bar{\Omega}$ and $f$ is the spatial distribution of the information on the support $D$. Then the output function (2) can be written in the following form

$$
z(t)=\int_{D} y(x, t) f(x) d x .
$$

A sensor may be pointwise (internal or boundary) if $D=\{b\}$ with $b \in \bar{\Omega}$ and $f=\delta(b-$.), where $\delta$ is the Dirac mass concentrated in $b$, and the sensor is then denoted by $\left(b, \delta_{b}\right)$. In this case the operator $C$ is unbounded and the output function (2) can be written in the following form

$$
z(t)=y(b, t) .
$$

We also recall that the system (1) together with the output (2) is said to be exactly (respectively weakly) observable in $\omega$ if $\operatorname{Im} \chi_{\omega} K^{*}=\mathrm{L}^{2}(\omega)$ (respective $\left.\overline{\operatorname{Im} \chi_{\omega} K^{*}}=\mathrm{L}^{2}(\omega)\right)$ For more details, we refer the reader to (see [1]).

Let $\alpha($.$) and \beta($.$) be two functions defined in$ $\mathrm{L}^{2}(\omega)$ such that $\alpha(.) \leq \beta($.$) a.e. in \omega$. Throughout the paper we set

$$
[\alpha(.), \beta(.)]=\left\{y \in \mathrm{L}^{2}(\omega) \mid \alpha(.) \leq y(.) \leq \beta(.) \text { a.e. in } \omega\right\}
$$

\section{Definition 2.1}

The system (1) together with the output (2) is said to be $[\alpha(),. \beta()$.$] -observable in \omega$ if

$$
\left(\operatorname{Im} \chi_{\omega} K^{*}\right) \bigcap[\alpha(.), \beta(.)] \neq \varnothing
$$

\section{Definition 2.2}

The sensor $(D, f)$ is said to be $[\alpha(),. \beta()$.$] -strategic in \omega$ if the observed system is $[\alpha(),. \beta()$.$] -observable in \omega$.

\section{Remark 2.1}

- If the system (1) together with the output (2) is $[\alpha(),. \beta()$.$] -observable in \omega_{1}$ then it is $[\alpha(),. \beta()$.$] -observable in any subregion \omega_{2} \subset \omega_{1}$.

- If the system (1) together with the output (2) is exactly observable in $\omega$ then it is $[\alpha(),. \beta()$.$] .$ observable in $\omega$.

\section{Proposition 2.3}

We have the equivalence between the following statements.

1. The system (1) together with the output (2) is $[\alpha(),. \beta()$.$] - observable in \omega$.

2. $\left(\operatorname{ker} K \chi_{\omega}^{*}\right) \cap[\alpha(),. \beta()]=.\{0\}$.

\section{Proof.}

(1) $\Rightarrow$ (2)

We shall show that

$$
\begin{aligned}
& \left(\operatorname{Im} \chi_{\omega} K^{*}\right) \cap[\alpha(.), \beta(.)] \neq \varnothing \\
\Rightarrow & \operatorname{Ker}\left(K \chi_{\omega}^{*}\right) \cap[\alpha(.), \beta(.)]=\{0\}
\end{aligned}
$$

Suppose that

$$
\operatorname{Ker}\left(K \chi_{\omega}^{*}\right) \cap[\alpha(.), \beta(.)] \neq\{0\}
$$

Let's consider

$$
y \in \operatorname{Ker}\left(K \chi_{\omega}^{*}\right) \cap[\alpha(.), \beta(.)],
$$

such that

$$
y \neq 0 \text {. }
$$

Then

$$
y \in \operatorname{Ker}\left(K \chi_{\omega}^{*}\right)
$$

and

$$
y \in[\alpha(.), \beta(.)]
$$

We have

$$
\operatorname{Ker}\left(K \chi_{\omega}^{*}\right)=\operatorname{Im}\left(\chi_{\omega} K^{*}\right)^{\perp},
$$

thus

$$
y \in \operatorname{Im}\left(\chi_{\omega} K^{*}\right)^{\perp}
$$


such that

$$
y \neq 0 \text {. }
$$

Therefore

$$
y \notin \operatorname{Im}\left(\chi_{\omega} K^{*}\right)
$$

Then

$$
\operatorname{Ker}\left(K \chi_{\omega}^{*}\right) \cap[\alpha(.), \beta(.)] \subset C_{L^{2}(\omega)} \operatorname{Im}\left(\chi_{\omega} K^{*}\right) .
$$

Hence

$$
\begin{gathered}
\operatorname{Im}\left(\chi_{\omega} K^{*}\right) \subset \\
C_{L^{2}(\omega)} \operatorname{Ker}\left(K \chi_{\omega}^{*}\right) \cup C_{L^{2}(\omega)}[\alpha(.), \beta(.)] .
\end{gathered}
$$

We have

$$
\operatorname{Im}\left(\chi_{\omega} K^{*}\right) \subset C_{L^{2}(\omega)} \operatorname{Ker}\left(K \chi_{\omega}^{*}\right),
$$

accordingly

$$
\left(\operatorname{Im} \chi_{\omega} K^{*}\right) \cap \operatorname{Ker}\left(K \chi_{\omega}^{*}\right)=\varnothing,
$$

then

$$
\left(\operatorname{Im} \chi_{\omega} K^{*}\right) \bigcap\left(\operatorname{Im} \chi_{\omega} K^{*}\right)^{\perp}=\varnothing \quad \text { (Absurd). }
$$

Since

$$
\operatorname{Im}\left(\chi_{\omega} K^{*}\right) \subset C_{L^{2}(\omega)}[\alpha(.), \beta(.)],
$$

we have

$$
\left(\operatorname{Im} \chi_{\omega} K^{*}\right) \cap[\alpha(.), \beta(.)]=\varnothing \quad(\text { Absurd }) .
$$

Consequently

$$
\operatorname{Ker}\left(K \chi_{\omega}^{*}\right) \bigcap[\alpha(.), \beta(.)]=\{0\} .
$$

(2) $\Rightarrow(1)$

We shall show that

$$
\begin{aligned}
& \operatorname{Ker}\left(K \chi_{\omega}^{*}\right) \cap[\alpha(.), \beta(.)]=\{0\} \\
& \Rightarrow \operatorname{Im}\left(\chi_{\omega} K^{*}\right) \cap[\alpha(.), \beta(.)] \neq \varnothing
\end{aligned}
$$

Suppose that

$$
\operatorname{Ker}\left(K \chi_{\omega}^{*}\right) \cap[\alpha(.), \beta(.)]=\{0\} .
$$

Let's consider

$$
y \in \operatorname{Ker}\left(K \chi_{\omega}^{*}\right) \bigcap[\alpha(.), \beta(.)] .
$$

Then $y \in \operatorname{Ker}\left(K \chi_{\omega}^{*}\right)$ and $y \in[\alpha(),. \beta()$.$] ,$ such that $\mathrm{y}=0$. We have $\operatorname{Ker}\left(K \chi_{\omega}^{*}\right)=\operatorname{Im}\left(\chi_{\omega} K^{*}\right)^{\perp}$, so $y \in \operatorname{Im}\left(\chi_{\omega} K^{*}\right)^{\perp}$ such that $y=0$.

Hence

$$
y \in\left(\operatorname{Im} \chi_{\omega} K^{*}\right) \text { and } y \in[\alpha(.), \beta(.)] .
$$

Thus

$$
\left(\operatorname{Im} \chi_{\omega} K^{*}\right) \bigcap[\alpha(.), \beta(.)] \neq \varnothing .
$$

Which shows that the system (1) together with the output (2) is $[\alpha(),. \beta()$.$] - observable in \omega$.

\section{HUM Approach}

The purpose of this section is to present an approach that allows the reconstruction of the initial state between the two functions $\alpha($.) and $\beta($.) into $\omega$. The approach constitutes an extension of the Hilbert Uniqueness Method developed by Lions (see [7]) to the case of the regional enlarged observability. Let the initial state decomposed in the following form

$$
y_{0}= \begin{cases}y_{0}^{1} & \text { in }[\alpha(.), \beta(.)] \\ y_{0}^{2} & \text { in } \mathrm{L}^{2}(\Omega) \backslash[\alpha(.), \beta(.)] .\end{cases}
$$

In the sequel our object is the reconstruction of the component $y_{0}^{1}$ in $[\alpha(),. \beta()$.$] , let G$ be defined by

$G=\left\{g \in L^{2}(\Omega) \mid g=0\right.$ in $\left.L^{2}(\Omega) \backslash[\alpha(),. \beta()].\right\}$

Let us go further in the state reconstruction by considering various types of sensors.

\subsection{Pointwise Sensors}

In this case, the output function is given by

$$
z(t)=y(t, b),
$$

where $b \in \Omega$ denote the given location of the sensor. 
For $\varphi_{0} \in G$, we consider the following system.

$$
\left\{\begin{array}{llll}
\frac{\partial \varphi(x, t)}{\partial t}=A \varphi(x, t) & \text { in } & Q \\
\varphi(x, 0) & =\varphi_{0}(x) & \text { in } & \Omega \\
\varphi(\xi, t)=0 & \text { on } & \Sigma .
\end{array}\right.
$$

We defined a semi-norm on $G$ by

$$
\left\|\varphi_{0}\right\|_{G}=\left[\int_{0}^{T} \varphi^{2}(t, b) d t\right]^{\frac{1}{2}},
$$

and we consider the retrograde system

$$
\left\{\begin{array}{llr}
-\frac{\partial \psi(x, t)}{\partial t}=A^{*} \psi(x, t)+\varphi(b, t) \delta(x-b) & \text { in } & Q \\
\psi(x, T)=0 & \text { in } & \Omega \\
\frac{\partial \psi(\xi, t)}{\partial v_{A^{*}}}=0 & \text { on } & \Sigma .
\end{array}\right.
$$

Let the operator $\Lambda$ be defined by

$$
\Lambda \varphi_{0}=P(\psi(0)),
$$

Where $P=\chi_{\omega}^{*} \chi_{\omega}$ and $\psi(0)=\psi(x, 0)$.

Consider the system

$$
\left\{\begin{array}{llr}
-\frac{\partial \bar{z}(x, t)}{\partial t}=A^{*} \bar{z}(x, t)+z(t) \delta(x-b) & \text { in } & Q \\
\bar{z}(x, T)=0 & \text { in } & \Omega \\
\overline{\frac{\partial z(\xi, t)}{\partial v_{A^{*}}}}=0 & \text { on } & \Sigma .
\end{array}\right.
$$

If $\varphi_{0}$ is chosen such that $\bar{z}(0)=\psi(0)$ in $\omega$, then the system (12) can be seen as an adjoint of the system (1) and our problem of the enlarged observability is to solve the equation

$$
\Lambda \varphi_{0}=P(\bar{z}(0)) \text {. }
$$

\section{Proposition 3.1}

If the system (1) together with the output (2) is $[\alpha(),. \beta()$.$] -observable in \omega$, then the equation (13) admits a unique solution $\varphi_{0} \in G$, which coincides with the initial condition $y_{0}^{1}$ to be observed in $[\alpha(),. \beta()$.$] .$

\section{Proof.}

1. Firstly, we show that if the system (1) together with the output (2) is $[\alpha(),. \beta()$.$] -observable in \omega$, then (9) defines a norm on $G$.

Let's consider $\varphi_{0} \in G$, we have

$\left\|\varphi_{0}\right\|_{G}=0 \Rightarrow \varphi(b, t)=0$ for all $t \in[0, T]$.

Or

$$
K \chi_{\omega}^{*} \chi_{\omega} \varphi_{0}=\mathrm{CS}(.) \chi_{\omega}^{*} \chi_{\omega} \varphi_{0}=0 .
$$

Hence

$$
\chi_{\omega} \varphi_{0} \in \operatorname{Ker}\left(K \chi_{\omega}^{*}\right)
$$

For

$$
\chi_{\omega} \varphi_{0} \in[\alpha(.), \beta(.)]
$$

then

$$
\chi_{\omega} \varphi_{0} \in \operatorname{Ker}\left(K \chi_{\omega}^{*}\right) \bigcap[\alpha(.), \beta(.)]
$$

and as the system is $[\alpha(),. \beta()$.$] -observable in \omega$, hence $\chi_{\omega} \varphi_{0}=0$. Consequently $\varphi_{0}=0$. Then (9) is a norm.

2. Let $\widehat{G}$ be the completion set of $G$ with respect to the norm (9) equipped with the associated inner product $\langle., .\rangle_{\widehat{G}}$ and $\widehat{G}^{*}$ its dual. We show that $\Lambda$ is an isomorphism from $\widehat{G}$ into $\widehat{G}^{*}$.

On the other hand, multiplying (10) by $\varphi$ (the solution to (8)) and integrating the result by part, we obtain

$$
\begin{aligned}
\int_{Q}-\varphi \psi^{\prime} d t d x & =\int_{Q} \varphi A^{*} \psi d t d x \\
& +\int_{Q} \varphi \varphi(b, t) \delta(x-b) d t d x
\end{aligned}
$$

Then 
$-\int_{\Omega}[\varphi \psi]_{0}^{T} d x+\int_{Q} \varphi^{\prime} \psi d t d x=\int_{Q} \varphi A^{*} \psi d t d x+\int_{0}^{T} \varphi^{2}(b, t) d t$.

Therefore

$$
\begin{aligned}
& \int_{\Omega}(\varphi(0) \psi(0)-\varphi(T) \psi(T)) d x= \\
& \int_{Q}\left(\varphi A^{*} \psi-\psi A \varphi\right) d t d x+\int_{0}^{T} \varphi^{2}(b, t) d t .
\end{aligned}
$$

Using Green formula, we have

$$
\begin{aligned}
\int_{\Omega} \varphi(0) \psi(0) d x & =\int_{\Sigma} \varphi \frac{\partial \psi}{\partial v_{A^{*}}} d \Sigma-\int_{\Sigma} \psi \frac{\partial \varphi}{\partial v_{A}} d \Sigma \\
& +\int_{0}^{T} \varphi^{2}(b, t) d t .
\end{aligned}
$$

Thus

$$
\int_{\Omega} \varphi(0) \psi(0) d x=\int_{0}^{T} \varphi^{2}(b, t) d t .
$$

Hence

$$
\left\langle\Lambda \varphi_{0}, \varphi_{0}\right\rangle=\int_{0}^{T} \varphi^{2}(b, t) d t
$$

Then

$$
\left\langle\Lambda \varphi_{0}, \varphi_{0}\right\rangle=\left\|\varphi_{0}\right\|_{G}^{2}
$$

We deduce that $\Lambda: \widehat{G} \rightarrow \widehat{G}^{*}$ is an isomorphism, then the equation (13) has a unique solution $\varphi_{0} \in G$ which corresponds to the initial state observed in $[\alpha(),. \beta()$.$] .$

\subsection{Zonal Sensors}

Let us come back to the system (1) and suppose that the measurements are given by an internal zone sensor defined by $(D, f)$, with $D \subset \Omega$ and $f \in L^{2}(D)$. The system is augmented with the output function

$$
z(t)=\int_{D} y(x, t) f(x) d x .
$$

In this case, we consider the system (8), $G$ be given by (6), and we defined a semi-norm on $G$ by

$$
\left\|\varphi_{0}\right\|_{G}=\left[\int_{0}^{T}\langle\varphi(t), f\rangle_{L^{2}(\Omega)}^{2} d t\right]^{\frac{1}{2}},
$$

with the system

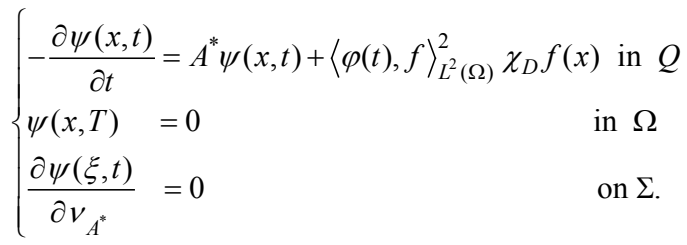

We introduce the operator

$$
\Lambda \varphi_{0}=P(\psi(0)) \text {, }
$$

where $P=\chi_{\omega}^{*} \chi_{\omega}$ and $\psi(0)=\psi(x, 0)$.

Consider the system

$$
\left\{\begin{array}{lrlrl}
-\frac{\partial \bar{z}(x, t)}{\partial t}=A^{*-} \bar{z}(x, t)+z(t) \chi_{D} f(x) & & \text { in } & Q \\
\bar{z}(x, T) & =0 & & \text { in } & \Omega \\
\frac{\partial \bar{z}(\xi, t)}{\partial v_{A^{*}}}=0 & \text { on } & \Sigma .
\end{array}\right.
$$

If $\varphi_{0}$ is chosen such that $\bar{z}(0)=\psi(0)$ in $\omega$, then the system (18) can be seen as an adjoint of the system (1) and our problem of the enlarged observability is to solve the equation

$$
\Lambda \varphi_{0}=P(\bar{z}(0)) .
$$

\section{Proposition 3.1}

If the system (1) together with the output (2) is $[\alpha(),. \beta()$.$] -observable in \omega$, then the equation (19) admits a unique solution $\varphi_{0} \in G$, which coincides with the initial condition $y_{0}^{1}$ to be observed in $[\alpha(),. \beta()$.$] .$

\section{Proof.}

The proof is similar to the pointwise case.

\section{Numerical Approach}

We have seen that the regional enlarged observability 
is equivalent, in all cases, to solving the equation

$$
\Lambda \varphi_{0}=P(\bar{z}(0)) \text {. }
$$

The numerical approximation of (20) is realized when one can have a basis $\left(\widetilde{\varphi_{i}}\right)$ of $\mathrm{L}^{2}(\Omega)$ and the idea is to calculate the components $\Lambda_{i j}$ of the operator $\Lambda$. Then we approximate the solution of (20) by the linear system

$$
\sum_{j=1}^{N} \Lambda_{i j} \varphi_{0, j}=\bar{z}_{i} \text { for } i=1, \ldots, N
$$

where $N$ is the order of approximation and $\bar{z}_{i}$ are the components of $P(\bar{z}(0))$ in the basis $\left(\widetilde{\varphi_{i}}\right)$ considered. Assume that $\left(\widetilde{\varphi_{i}}\right)$ is the set of the eigenfunctions of the operator $A$ associated with the eigenvalues $\lambda_{i}$ of multiplicity one. In this case of pointwise measurements, we have

$$
\begin{aligned}
\left\langle\Lambda \varphi_{0}, \varphi_{0}\right\rangle= & \sum_{i, j=1}^{\infty}\left\langle\tilde{\varphi}_{i}, \varphi_{0}\right\rangle_{\mathrm{L}^{2}(\omega)}\left\langle\tilde{\varphi}_{j}, \varphi_{0}\right\rangle_{\mathrm{L}^{2}(\omega)} \\
& \times \frac{-1+e^{\left(\lambda_{i}+\lambda_{j}\right) T}}{\lambda_{i}+\lambda_{j}} \tilde{\varphi}_{i}(b) \tilde{\varphi}_{j}(b)
\end{aligned}
$$

Then the components of $\Lambda$ are given by

$$
\Lambda_{i j}=\frac{-1+e^{\left(\lambda_{i}+\lambda_{j}\right) T}}{\lambda_{i}+\lambda_{j}} \tilde{\varphi}_{i}(b) \tilde{\varphi}_{j}(b)
$$

In the zonal case, the same developments lead to the components of $\Lambda$

$$
\Lambda_{i j}=\frac{-1+e^{\left(\lambda_{i}+\lambda_{j}\right) T}}{\lambda_{i}+\lambda_{j}}\left\langle f, \tilde{\varphi}_{i}\right\rangle_{\mathrm{L}^{2}(D)}\left\langle f, \tilde{\varphi}_{j}\right\rangle_{\mathrm{L}^{2}(D)}
$$

Algorithm. In the case of pointwise sensor, we have the following algorithm:

Step 1:

- The subregion $\omega$, the location of the sensor $b$.

- Choose the function $y_{0} \in[\alpha(),. \beta()$.$] .$

- Threshold accuracy $\varepsilon$.
Step 2: Repeat

$\triangleright$ Slove the system (8) to obtain $\varphi$.

Slove the system (12) to obtian $\bar{z}$.

Solve the equation (21) to obtain $\varphi_{0}$.

Until $\left\|y_{0}-\varphi_{0}\right\|_{L^{2}(\omega)}^{2} \prec \varepsilon$.

Step 3: The solution $\varphi_{0}$ corresponds to the initial state to be observed in $[\alpha(),. \beta()$.$] .$

\section{Simulation Results}

Here, we present a numerical example which illustrates the previous algorithm.

The obtained results are related to the initial condition and the sensor location. Let's consider the following one-dimensional system in $\Omega=] 0,1[$ excited by a pointwise sensor

$$
\left\{\begin{array}{lll}
\frac{\partial y(x, t)}{\partial t}=\frac{\partial y^{2}(x, t)}{\partial x^{2}} & \text { in } & \Omega \times] 0, T[ \\
y(0, t)=y(1, t)=0 & \text { in } & ] 0, T[ \\
y(x, 0)=y_{0}(x) & \text { in } & \Omega,
\end{array}\right.
$$

augmented with the output function

$$
z(t)=y(b, t), b \in \Omega .
$$

The initial state to be reconstructed is

$$
y_{0}(x)=\frac{1}{2}\left[x^{2}(x-1)^{2}-2 x(x-1)\right]
$$

Let

$$
\begin{gathered}
\alpha(x)=-2 x(x-1)\left(x-\frac{3}{4}\right) \\
\beta(x)=-2 x(x-1)
\end{gathered}
$$

Applying the previous algorithm for $\omega=] 0.5,0.65[$ and $b=0.85$ (location of the sensor), we obtain the following results:

If the sensor is located in $b=0.98$, then we obtain 


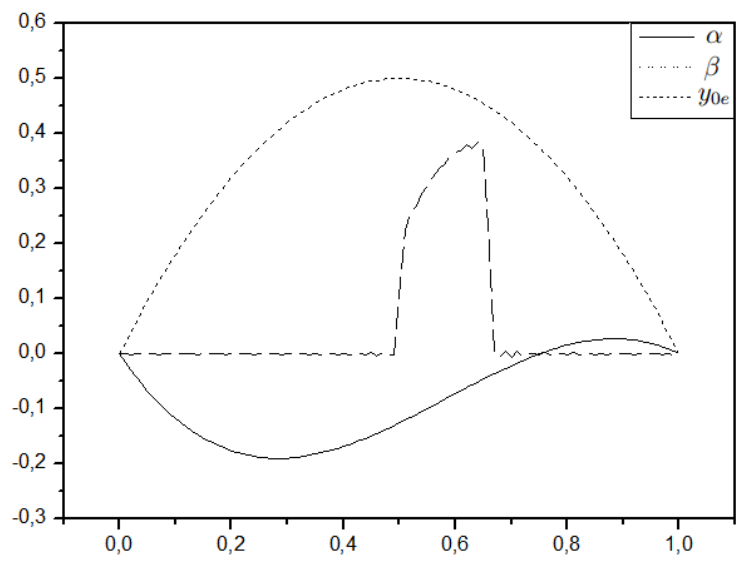

Fig. 2 The estimated initial state $y_{0 e}$.

Figure. 2 shows that the sensor located in $\mathrm{b}=0.85$ is $[\alpha(),. \beta()$.$] -strategic in \omega=] 0.5,0.65[$ with the error $\varepsilon=2.97 \times 10^{-2}$.

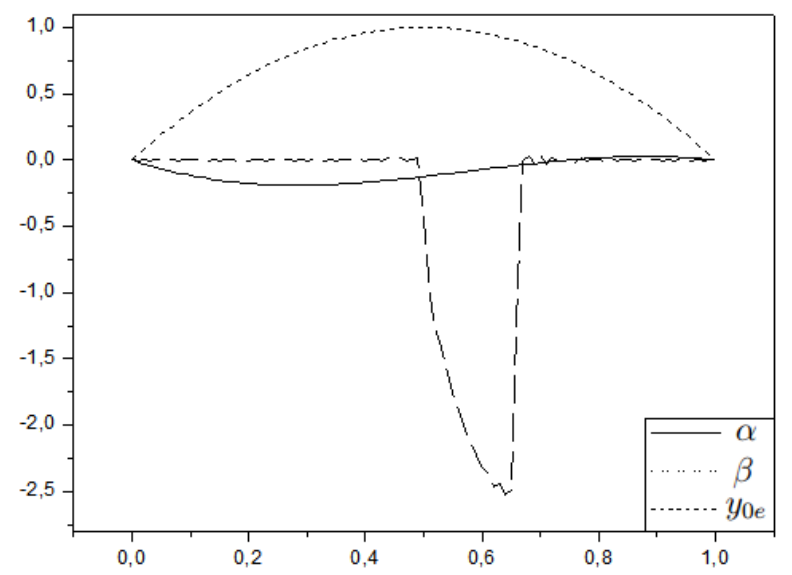

Fig. 3 The estimated initial state $y_{0 e}$.

Figure. 3 shows a non-strategic position of the sensor and then the estimated initial state is not between $\alpha($.$) and \beta($.$) in \omega$.

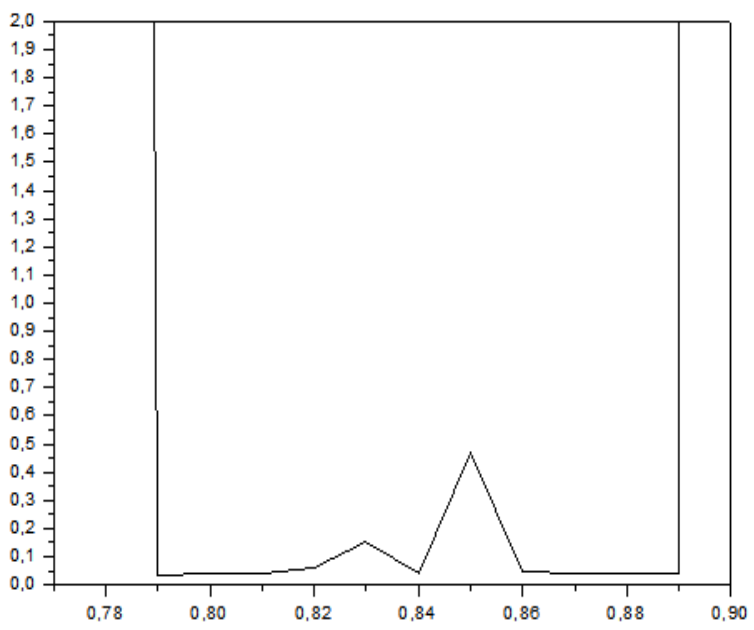

Fig. 4 The reconstruction error with respect to the sensor location b.

We remark that the positions in $[0,0.7]$ and $[0,0.91]$ are not-strategic. The Figure. 4 shows the best positions of the sensors location $b$.

\section{Conclusions}

The question of enlarged observability for distributed linear parabolic systems was discussed and solved using the Hilbert Uniqueness Method. We explored a useful numerical approach which allows the computation of the obtained algorithm and which is illustrated by numerical example and simulations. The case where $\omega$ is part of the boundary $\partial \Omega$ of the system evolution domain $\Omega$ is under consideration and the work will be the subject of a future paper.

\section{Acknowledgment}

This work has been carried out with a grant from Hassan II Academy of Sciences and Technology.

\section{References}

[1] M. Amouroux, A. EL Jai and E. Zerrik, Regional observability of distributed systems, International Journal of Systems Science, 1994, Vol 25, 301-313.

[2] R.F. Curtain and A. J. Pritchard, Infinite dimensional linear systems theory, Springer, Lecture Notes in Control and Informations, Science, Springer, 1978, New York.

[3] R.F. Curtain and H. Zwart, An introduction to infinite dimensional linear systems theory, Texts in Applied 
Mathematics, 1995, Vol 21, Springer Verlag.

[4] A. EL Jai, M.C Simon and E. Zerrik, Regional observability and sensors structures, Sensors and Actuators Journal, 1993, Vol 39, 95-102.

[5] A. EL Jai and A.J. Pritchard, Sencors and actuators in distributed systeme analysis, Ellis Horwood Series in Applied Mathematics, 1988, J. Wiley.

[6] J.L. Lions and E. Magenes, Problèmes aux limites non homogènes et applications, 1968, Vol 1 et 2, Dunod, paris.

[7] J.L. Lions, Contrôlabilité exacte perturbations et stabilisation des systèmes distribués, Tome 1 , contrôlabilité exacte, 1988, Masson, Paris.

[8] J.L. Lions, Sur la contrôlabilité exacte élargie, Progress in Nonlinear Differential Equations and Their Applications, 1989, Vol 1, 703-727.

[9] L. Badraoui, A. Boutoulout and H. Bourray, Controllability of distributed parameter bioreactor: wastewater equation, International Journal of Computation and Numerical Analysis and Application, 2004, Vol 5, No 3, 261-279.

[10] E. Zerrik, H. Bourray and A. Boutoulout, Regional boundary observability: A numerical approach, Int. J. Appl. Math. Compat. Sci, 2002, Vol 12, No 35, 143-151. 